\title{
Commentary: PREMM5 threshold of $2.5 \%$ is recommended to improve identification of PMS2 carriers
}

\author{
Fay Kastrinos ${ }^{1} \cdot$ Hajime Uno $^{2} \cdot$ Sapna Syngal ${ }^{2,3}$ \\ Published online: 29 January 2018 \\ ๑) Springer Science+Business Media B.V., part of Springer Nature 2018
}

We appreciate the opportunity to comment on the recently published article by Goverde et al. entitled, "Evaluation of current prediction models for Lynch syndrome: updating the PREMM5 model to identify PMS2 mutation carriers" [1].

We concur with the findings that the $\mathrm{PREMM}_{5}$ model's ability to discriminate carriers of pathogenic PMS2 gene mutations from noncarriers is less robust than for the other Lynch syndrome associated genes at a threshold of $\geq 5 \%$, including $M L H 1, M S H 2$, and MSH6. While the authors report an AUC of 0.51 for PREMM $_{5}$ 's ability to discern $P M S 2$ carriers from noncarriers, we caution the interpretation of these results due to the clinic-based cohort in which the results were derived, the associated prevalence of mismatch (MMR) gene mutations, and the small number $(\mathrm{n}=12)$ of $P M S 2$ carriers from which the analyses were conducted to assess PREMM $_{5}$ 's performance, leading to large uncertainty on the predictive performance. While our results also showed less optimal discrimination of PMS2 from noncarriers with an AUC of 0.64 , the derivation cohort was comprised of 141 PMS2 mutation carriers [2]. We therefore encourage further validation of gene-specific evaluation of the PREMM5 model to include a larger sample of mutation carriers, particularly those with pathogenic PMS2 gene variants.

We hope that additional studies and collaborative efforts will consider the following elements in the study design. First, we recommended use of a new threshold of $\geq 2.5 \%$ to guide Lynch syndrome testing [2]. At this threshold, the

Fay Kastrinos

fk18@columbia.edu

1 Herbert Irving Comprehensive Cancer Center, Division of Digestive and Liver Diseases, Columbia University Medical Cancer, New York, NY, USA

2 Population Sciences Division, Dana-Farber Cancer Center, Boston, MA, USA

3 Division of Gastroenterology, Brigham and Women's Hospital, Harvard Medical School, Boston, MA, USA model's sensitivity improved, maintained a high negative predictive value and decreased the overall number needed to test in order to identify one mutation carrier albeit with a decrease in specificity. Additional studies that specifically assess the newly recommended cutoff value of $\geq 2.5 \%$ are needed. Second, we recommend use of the PREMM model for overall prediction of Lynch syndrome MMR genes, rather than gene-specific prediction. Gene specific evaluation for Lynch syndrome may be an option based on available resources at individual institutions that screen for Lynch syndrome, as current practices currently vary not only between institutions but countries as well, but performance of the model is expected to vary based on individual genes as discussed in our paper. Lastly, use of the PREMM model is valuable particularly in individuals without a personal history of cancer but a relevant family history of cancer. While the authors have improved the performance of $\mathrm{PREMM}_{5}$ with the inclusion of colorectal cancer location as an additional predictor, use of the expanded PREMM ${ }_{5}$ model would be limited to only those with a diagnosis of colorectal cancer.

Additional studies that further validate the $\mathrm{PREMM}_{5}$ model's performance in diverse population settings, including those unaffected by cancer would be valuable. We continue to update the PREMM model as the field of clinical cancer genetics continues to evolve and appreciate collaborative efforts to further improve the identification of individuals with Lynch syndrome.

\section{References}

1. Goverde A, Spaander MCW, Nieboer D et al (2017) Evaluation of current prediction models for Lynch syndrome: updating the PREMM5 model to identify PMS2 mutation carriers. Fam Cancer. https://doi.org/10.1007/s10689-017-0039-1 [Epub ahead of print]

2. Kastrinos F, Uno H, Ukaegbu C et al (2017) Development and validation of the PREMM5 model for comprehensive risk assessment of lynch syndrome. J Clin Oncol 35(19):2165-2172 